4

5

6

\title{
The contribution of whole-genome sequencing to our understanding of the epidemiology and control of methicillin-resistant Staphylococcus aureus
}

\author{
Hilary Humphreys ${ }^{\mathrm{a}, \mathrm{b}}$, David C. Coleman ${ }^{\mathrm{c}}$
}
a Department of Clinical Microbiology, the Royal College of Surgeons in Ireland and the ${ }^{\mathrm{b}}$ Department of Microbiology, Beaumont Hospital, Dublin Ireland.
${ }^{c}$ Microbiology Research Unit, Dublin Dental University Hospital, Trinity College Dublin, University of Dublin, Dublin, Ireland

Address and contact details: $\quad$ Department of Clinical Microbiology

RCSI Education and Research Centre

Beaumont Hospital

Dublin D09 YD 60, Ireland

'Phone + +35318093710/3708

Fax $\quad+35318092871$

Email $\quad$ hhumphreys@,rcsi.ie

Running title Whole genome sequencing and MRSA Control 


\section{Abstract}

28 In recent years, approaches to tracking the spread of methicillin-resistant Staphylococcus aureus

29 (MRSA) as part of outbreak management have used conventional DNA-based methods including 30 pulsed field gel electrophoresis (PFGE) and spa typing. However, when a predominant clone is present, these methods may be insufficiently discriminatory. We conducted a literature search to highlight how whole genome sequencing (WGS) has revolutionised the investigation of outbreaks of MRSA, including intra-hospital spread and MRSA in the community, and to review its future potential. Whole genome sequencing provides enhanced isolate discrimination, as it permits the entire genomic DNA sequence of isolates to be rapidly determined and compared. Many software packages used for the analysis of WGS data are becoming increasingly available. To date WGS has been more sensitive in confirming outbreaks, often persisting for prolonged periods, previously undetected by conventional molecular typing. The evolving dynamic of spread from the community to hospitals, within and between hospitals, and from hospitals to the community, is only becoming clear with WGS studies, and is more complex and convoluted than widely appreciated. Also, WGS can exclude cross-transmission, when isolates are different. The challenges now are to make WGS technology more amenable for routine use and to develop an evidence-based consensus for sequence difference thresholds for isolates that they are deemed part of the same outbreak, including protracted outbreaks. Using such data in a timely way will provide increased sensitivity in detecting cross-transmission events earlier with the potential of preventing outbreaks to positively impact on infection prevention and control.

Keywords: Whole genome sequencing, MRSA, bioinformatics, MRSA clones, outbreaks, spread, acute hospitals, community, long stay residential units. 
67

68

\section{Background}

Staphylococcus aureus is a common cause of healthcare-associated infection (HCAI). In a recent European study in children, S. aureus was responsible for $11 \%$ of infections and was second only to coagulase-negative staphylococci as a cause [1]. In a four-country prevalence survey of HCAI in the UK and Ireland, methicillin-resistant $S$. aureus (MRSA) was responsible for $15.8 \%$ of all HCAIs [2]. In addition to causing serious infection in acutely ill patients in hospital, MRSA can spread to and between residents in long stay residential units (LSRU) and day units, as increasingly the elderly population is managed outside acute hospitals.

Infections caused by MRSA have been prevalent in many countries for decades even if in recent years, the prevalence of some serious infections, such as bloodstream infections (BSI), have declined $[3,4]$. Data from the UK and Northern Ireland from 2010-2014 derived from mandatory reporting of $S$. aureus bloodstream infection has shown that the proportion of BSI due to $S$. aureus has fallen from $16.2 \%$ to $8.9 \%$ with a downward trend in all four countries [3]. Molecular typing is important to track spread, indicate the possible origins of outbreaks, and confirm the efficacy of outbreak control measures and track the emergence or evolution of new clones in the outbreak and endemic setting.

\section{Conventional molecular typing of MRSA}

Routes of MRSA transmission and spread in healthcare settings have traditionally been investigated using targeted DNA-based typing methods including pulse-field gel electrophoresis (PFGE), spa typing, conventional multilocus sequence typing (MLST), and more recently, the application of DNA microarry technology $[5,6,7]$. In many cases these approaches provide valuable insights into the relatedness of clinical isolates when combined with relevant 
epidemiological data and the expertise and insights of infection prevention and control staff. However, conventional molecular typing approaches frequently struggle to discriminate between isolates in the healthcare setting where a particular or limited number of MRSA clonal lineages predominate. In many countries MRSA responsible for HCAIs frequently belong to a relatively small number of clones; for example, in Ireland and the UK multilocus sequence type (ST) 22 has predominated among MRSA responsible for bloodstream infections and carriage for almost two decades [7-9]. Isolates belonging to this lineage are particularly recalcitrant to discrimination by conventional molecular typing approaches, making detection of all or even major patterns of spread problematic $[7,10,11]$. Consequently, there is a need for more discriminatory methods to distinguish clonal isolates to track spread.

\section{Brief overview of whole genome sequencing of nosocomial pathogens}

\section{Next generation sequencing}

Table 1 provides a list and explanations of commonly used terms relating to WGS and its analysis. Over the last decade the development of next generation sequencing (NGS) to determine the entire genetic sequence of microbial pathogens, especially bacterial species, has revolutionised molecular epidemiology. In contrast to Sanger sequencing, NGS permits the highthroughput and rapid determination of whole genome sequences of pathogens at an affordable cost. A variety of NGS approaches have been developed that utilise innovative sequencing chemistry methods in tandem with small footprint automated bench top sequencers [12-15]. These approaches involve fragmentation of genomic DNA into short segments of a few hundred nucleotide bases in length, tagging of the fragments with adapters to generate genome fragment libraries, immobilisation of the libraries on a solid interface, followed by PCR amplification of 
97 the DNA fragments. The DNA sequence of each fragment is determined during complementary strand synthesis using a variety of approaches depending on the NGS platform being used. This allows millions to billions of DNA fragments to be sequenced in parallel.

Two of the most widely used NGS platforms, also known as second generation sequencers or 102 short read sequencers, include the Illumina sequencing systems (Illumina, Eindhoven, The Netherlands) and the ion semiconductor systems (Thermo Fisher Scientific) $[12,13,16]$. In recent 104 years, most studies reporting NGS of bacterial pathogens have used Illumina short read sequencers. In contrast, third generation DNA sequencers yield much longer sequencing reads

106 (i.e. $>10 \mathrm{~kb}$ ) and can sequence single DNA molecules without the requirement for DNA 107 amplification. Examples of third generation sequencing platforms include the Single Molecular 108 Real-Time (SMRT) sequencing platforms manufactured by PacBio (Menlo Park, CA, USA) and 109 the nanopore MinION sequencers manufactured by Oxford Nanopore Technologies (Oxford UK) 110 [12]. The error rates generated by third generation sequencers are relatively high compared with 111 second-generation sequencers, but the long-read lengths generated by the former are very 112 advantageous for sequencing entire genomes and especially for plasmids. However, PacBio 113 platforms are currently relatively expensive and are not suitable for the high-throughput rapid 114 analysis or the processing of many clinical isolates. In contrast, Oxford Nanopore sequencers are 115 inexpensive and can yield very long sequence reads, but bioinformatics analysis of sequence data 116 is more challenging. Nonetheless, a growing range of nanopore bioinformatics tools are being 117 developed, which soon will make this technology more readily applicable to clinical isolates 118 [17]. A combination of short read sequences generated by NGS platforms such as Illumina 119 MiSeq and long read sequences generated by nanopore sequencers can be used to rapidly and 
120 accurately map relatively large genomic regions such as the staphylococcal cassette chromosome

121 mec $(\mathrm{SCCmec})$ region and other large genomic elements such as the arginine catabolic mobile

122 element (ACME) in S. aureus and coagulase-negative staphylococcal species. Typically, the

123 DNA sequences of such elements are spread across several contiguous sequences in genome

124 assemblies generated from short read sequences and usually require extensive PCRs to

125 accurately refine the assemblies. This can be overcome by generating hybrid assemblies of both

126 short read and long read sequences [18,19]. A number of recent reviews have provided

127 comprehensive overviews of whole genome sequencing (WGS) platforms and technology and its

128 applications for microbial epidemiology [12-15].

Genome assembly and bioinformatics

131 The volume and complexity of data generated by WGS platforms requires the application of a 132 variety of bioinformatics tools to determine the quality of the sequence data and to transform 133 unrefined sequencing read data into more useful or meaningful forms. Software algorithms are 134 used to clean up and organise sequence data, to assemble genome sequences from overlapping 135 sequence reads, and to identify genomic variants, for genotyping and for phylogenetic analysis. 136 For the non-bioinformatician, the terminology and application of bioinformatics is daunting in 137 this rapidly developing field. A recent review by Carriço et al. (2018) provides an excellent 138 overview of bioinformatics as applied to WGS data for the non-expert [20]. Some of the more 139 frequently used bioinformatics approaches and software for microbial genome assembly and 140 subsequent analysis have been recently reviewed [20,21,12]. Many software packages used for 141 the analysis of WGS data are freely available and several commercially available and easy to use 
142 software packages including BioNumerics (Applied Maths, Ghent, Belgium) and SeqSphere

143 (Ridom GmBH, Münster, Germany) are widely used for this purpose $[12,14,16]$

144 Applications of WGS data for epidemiology

145 Whole genome sequencing data can be used for a variety of purposes in investigating outbreaks 146 of infection and in tracking the sources and spread of infection in hospitals as well as in

147 investigating more regional and global aspects of the emergence of specific clones of particular 148 pathogens. Conventional targeted molecular typing of pathogens (e.g. PFGE or conventional 149 MLST) generates a genotype barcode or molecular fingerprint of each isolate based on a 150 relatively small portion of the genome $[7,12,14]$. Isolates with identical or similar genotypes 151 linked by epidemiological data are presumed to represent linked cases of infection. In contrast, 152 WGS enables the entire genome of isolates to be compared, which significantly enhances 153 resolution. As with conventional molecular typing, the genomes of isolates recovered from an 154 outbreak or cluster of infections are likely to be closely related.

Single nucleotide variation analysis

157 All microorganisms accumulate changes in their genomes over time, often through random 158 mutations. These include alterations to single nucleotide bases (single nucleotide variations or 159 SNVs). When an SNV becomes fixed within a population it is referred to as a single nucleotide 160 polymorphism or SNP. Other types of changes to the genome include gene acquisition by 161 horizontal transfer (e.g. plasmid acquisition or lysogenisation with a bacteriophage), small 162 insertions and deletions, gene duplication and genome rearrangements.

164 SNV analysis is frequently used to type isolates based on WGS data in an outbreak scenario and 
165 has been used extensively using data generated by short read sequencers such as those 166 manufactured by Illumina [12-15, 22-24]. This approach involves mapping sequence reads or 167 larger contiguous sequences (known as contigs) assembled from overlapping short read 168 sequences to a reference genome by core genome alignment. Specific software filtering tools can 169 be used to exclude sequence stretches of high variability. Curated collections of reference 170 genomes for particular species (including S. aureus) are available for SNV analysis. However, 171 SNV analysis yields the best results when a reference genome that is closely related to the 172 samples under investigation is used. In the case of an outbreak, isolates are likely to be closely 173 related and one isolate sequence can be used as a reference against which other outbreak isolates'

174 sequences can be mapped. The alignment of isolate sequences can then be used for phylogenetic 175 analysis to determine the relationships between the isolates based on the identification of bases 176 that differ in the test samples relative to the corresponding bases in the reference genome.

Extended multilocus sequence typing

179 The genetic relatedness of isolates can also be investigated using extended versions of 180 conventional MLST. Conventional MLST typically involves the sequencing of segments of a 181 small number of selected housekeeping genes that accumulate genetic changes relatively slowly 182 because the encoded proteins are functionally constrained [25]. Such genes encode segments that 183 are amplified by PCR using specific primers and the amplimers sequenced by conventional 184 Sanger sequencing. Combinations of allelic variants in the selected genes are used to define a 185 sequence type (ST) for a particular microorganism. The advent of WGS has enabled the establishment of whole genome (wg) and core genome (cg) MLST schemes that enable a 187 comparison of test sequences with large curated sets of predefined genes for a particular species 
188 [26,27]. Publicly available curated gene sets can include hundreds to thousands of genes 189 depending on the scheme and analysis can be undertaken with easy to use software packages 190 such as BioNumerics and SeqSphere [14]. The use of curated cgMLST schemes facilitates good 191 interlaboratory reproducibility.

\section{Thresholds of isolate relatedness}

194 Establishing SNV and cgMLST thresholds of relatedness for a microorganism can be 195 problematic. Genomic variability increases over time and it is vital that this consideration is 196 borne in mind when attempting to assess the relatedness of isolates based on WGS data. There 197 are no definitive rules for determining isolate relatedness or what constitutes a significant 198 difference between isolates. Judgements on similarity or significantly different should be made 199 separately according to the facts of each case. Meaningful thresholds of relatedness can be 200 developed by investigating epidemiologically linked and unrelated isolates. However, proposed 201 thresholds should always be interpreted in conjunction with epidemiological data.

A recent study that investigated several outbreaks of $S$. aureus using WGS suggested that in an

204 acute short-term outbreak, there will be insufficient time for diversity to accumulate [23] and 205 therefore establishing thresholds in this scenario should be less problematic. Schürch et al. 206 (2018) recently detailed a list of current suggested SNV and cgMLST relatedness criteria for 207 some representative clinically significant bacterial species [14]. It is worth bearing in mind that clonality thresholds may vary within particular clones of a particular pathogen.

\section{The use of WGS for investigating MRSA outbreaks}


212 The more widespread availability of WGS in recent years has enabled us to study in more detail

213 patterns of spread, including detecting previously undocumented transmission, as well as the

214 overall and detailed evolution of strains of MRSA [28,29]. A study of Danish isolates using spa

215 typing and Sanger sequencing found a 97\% agreement between both these methods, and WGS

$216[30]$. As the technology becomes increasingly available and costs reduce, WGS will be no longer

217 confined to research or reference laboratory facilities but become increasingly more available in

218 routine clinical laboratories to inform infection prevention and control strategies, as well as

219 outbreak management in real time [30-32].

220

221 Price and colleagues in 2013 outlined the potential of WGS, discussed some recent applications

222 and highlighted its potential for the future [33]. We undertook to update that and highlight

223 important findings relating to the detection of outbreaks, their evolution over time, and inter-

224 hospital spread, and how this could potentially benefit preventative measures for MRSA in the

225 community. We did not set out to cover aspects of WGS and MRSA as they relate specifically to

226 antimicrobial resistance and global molecular epidemiology. A literature search was undertaken

227 of articles in PubMed, Embase, the Cochrane Library and Web of Science for articles up to the

228 end of August 2018. Search terms included MRSA, WGS, outbreaks, clinical, infection,

229 prevention and control. In total, 588 items were found, and with duplicates removed, and having

230 reviewed all titles and abstracts for relevance (e.g. excluding those related purely to the

231 veterinary setting), those cited focus on the value and relevance of WGS to MRSA in terms of

232 prevention and control and outbreak investigation. We excluded studies where the focus was

233 purely on clonal evolutionary trends and were not germane to infection prevention and control

234 practitioners. What follows is a discussion of the transmission of MRSA and especially outbreak 
management. Studies that refer to and or include methicillin-susceptible $S$. aureus (MSSA) are included were MRSA isolates were also included, and or, where the findings might equally apply to outbreaks or settings involving MSSA.

Young and colleagues have looked at the evolutionary dynamics of $S$. aureus from carriage to disease, i.e. a patient who carried $S$. aureus in the nose and who subsequently developed bloodstream infection (BSI) [34]. Just eight mutations accompanied the transmission from carriage to infection [34]. However, during an outbreak which occurs over a matter of weeks or even months, much of the literature seems to agree that up to approximately 30 SNPs may be allowed between isolates before the isolates are considered different, i.e. transmission may have occurred between two patients if their isolates differ by less than 30 SNPs $[11,14]$. Such conclusions are predicated on the assumption that the epidemiological findings are supportive.

\section{Epidemiology and control}

Neonatal and paediatric units

The occurrence of MRSA amongst neonates has potentially devastating consequences. Hence, many studies of WGS and MRSA have focussed on neonates or paediatric units, because of the serious clinical consequences. Whole genome sequencing has been used to track the spread of MRSA and to assist in early intervention measures [26, 35-43].

Köser and colleagues investigated a putative MRSA outbreak in the UK and WGS revealed a distinct cluster with clear separation between outbreak and non-outbreak isolates, amongst a collection of ST22 isolates [37]. When using less-discriminatory methods of typing, the extent of an outbreak may be exaggerated. In a neonatal unit outbreak involving 17 neonates, spa typing, 
259 PFGE and WGS were used. All 17 isolates belonged USA-300 isolates according to PFGE.

260 However, while five isolates were involved in recent transmission events, $12 \quad(70.5 \%)$

261 represented genetically unique isolates according to WGS and were therefore believed not to be

262 part of the outbreak [37]. This finding is important as it suggests that there was no obvious

263 deficiency in infection prevention measures by healthcare staff because there may be have been

264 multiple independent introductions of USA-300.

265

266 Earls and colleagues investigated two protracted outbreaks (2009-2011 and 2014-2017) in the

267 neonatal intensive care unit (NICU) of an Irish hospital involving clonal complex (CC) 88-

268 MRSA isolates belonging to spa types t186 and t786 [42]. Isolates were recovered from 20

269 separate neonates during the outbreaks, together with two isolates recovered two years apart

270 from the same healthcare worker. Whole genome sequencing and subsequent wgMLST analysis

271 revealed that both outbreaks were caused by the same CC88/ST78-MRSA-IVa strain. All the

272 isolates formed a large cluster, exhibiting 1-71 pairwise allelic differences in a wgMLST-based

273 minimum spanning tree (MST). The maximum distance observed between any two directly

274 linked nodes was 32 alleles, detected between two t186 isolates, which were recovered almost

275 three years apart during outbreak one. All other directly linked isolates exhibited 1-19 allelic

276 differences [42]. This indicated a high degree of relatedness between all isolates within the

277 cluster network. There were no apparent sub-clusters based on spa type and the one direct link

278 within the MST between spa type t786 isolates and spa type t186 isolates corresponded to an

279 allelic difference of 18 . The two t786 isolates recovered from a healthcare worker two years

280 apart exhibited 20 allelic differences and differed from other t786 isolates by 10-21 and 9-37

281 allelic differences, respectively, indicating the involvement of the HCW in the outbreak 
282 transmission. Unfortunately, no information on whether the HCW was persistently or transiently

283 colonised with the CC88/ST78-MRSA-IVa strain during the two year period was available or if 284 attempts to decolonise the HCW were undertaken. This study also demonstrated the spread of the 285 ST78-MRSA-IVa strain to two other Irish hospitals. A cgMLST-based comparison with 286 international comparator isolates showed that the outbreak strain was most likely imported from 287 Australia, where it is among the prevalent MRSA clones.

289 The French national staphylococcal reference laboratory used WGS to retrospectively investigate 290 MRSA isolates amongst four separate clonal complexes (CC1, CC5, CC8, CC30) involved in 291 community and hospital outbreaks, which included 41 CC5 isolates from new-borns [43]. Even 292 though the spa type was different, isolates from Limoges and Bordeaux, which are separated by 293 approximately $180 \mathrm{~km}$, differed by less than 22 SNPs suggesting that despite the geographical 294 difference, they could be part of the same transmission pattern [43]. The value of WGS in this 295 setting, are outlined in Table 2.

\section{Other hospital outbreaks}

298 Studies have shown the value of WGS in teasing out some of the subtleties of general and 299 hospital outbreak evolution and development $[11,16,23,44-50]$. The isolates from three separate 300 outbreaks were studied, including one in a hospital, resulting in a total of 42 isolates; 15 of 16 301 isolates from a burns unit formed a single cluster but 12 isolates from a post-surgical unit were 302 more diverse; the authors concluded that those with less than eight SNPs should be considered 303 related, and those between nine to 29 , as being possibly unrelated [16]. 
We have assessed the variability amongst MRSA isolates collected from patients and the environment in a prospective study that involved 41 patient and environmental isolates that were sequence type 22 [11]. We traced the isolates in terms of the geographical location and the time when they were recovered. Far more combinations of isolates, i.e. patient-patient or patientenvironment, indicating potential transmission links, were detected by WGS compared with conventional molecular typing using spa, dru and or PFGE typing or a combination of all three [11]. The $d r u$ region is a noncoding DNA segment consisting of imperfect 40-bp variablenumber tandem repeats (VNTRs) located in the hypervariable region between $m e c A$ and IS431mec of SCCmec [7,11].

In a study on a Dutch oncology ward involving an asymptomatic nasal colonized healthcare worker, WGS MLST showed similarities between MSSA and MRSA isolates involved in an outbreak and the authors hypothesised that a fusidic acid resistant isolate of MSSA acquired a SCCmec, and subsequently caused an MRSA outbreak suggested a genetic link [46]. Miller and colleagues were concerned about the patterns of MRSA BSIs in a specific hospital in England, involving a clonal variant of EMRSA-16. Isolates causing BSI between 2000 and 2001, and 2006 to 2007 were investigated [48]. The clonal variant was largely confined to that hospital unlike isolates causing BSIs acquired elsewhere, and infections caused by this clone were significantly associated with increased peripheral white cell and neutrophil count, suggesting increased virulence [48]. Similarly, a retrospective review of isolates in 2004-2014 was undertaken in Switzerland to reconstruct transmission pathways [49]. Tracking the geographic locations of patients who were colonised or infected together with WGS data, enabled the researchers to assess patterns of spread, which included a network of hospitals and overlapping periods of 
328 hospitalisation. In one case, an outbreak lasted several months in an orthopaedic ward, but was only retrospectively detected using WGS. Looking at isolates from the same patient over time, the authors concluded that there was one SNP every 8.9 weeks or 0.016 per day [50].

332 Tong et al. (2015) used WGS to investigate the genetic diversity of ST239 MRSA isolates from 333 patients over a three-month period in two ICU units of a 1000 bed hospital in Thailand where 334 transmission was common [22]. Phylogenetic analysis revealed a flux of distinct ST239 clades 335 (or groups of isolates) over time in each ICU. Analysis of WGS data confirmed intra-ward and 336 inter-ward transmission events and revealed that one patient in each ICU was the source of numerous transmission events. The mean pairwise SNP differences between the five ST239 clades identified was $\geq 197$ SNPs, indicating that each clade was distinctly different.

\section{Community and wider patterns of spread}

341 While the focus of the hospital infection prevention and control team is to largely prevent and 342 analyse outbreaks within the hospital, these may sometimes arise from outside the hospital, i.e. 343 from patients admitted from other hospitals or from LSRU as well as potentially from patients 344 who have been abroad, especially if hospitalised there. Hence, the analysis of a wider range of 345 isolates can inform preventative strategies and highlight the innate capacity of $S$. aureus to 346 spread and evolve.

348 The ST8 USA300 MRSA clone emerged shortly after 2000 and subsequently became the leading cause of skin and soft tissue infections in the United States (US). The origin of USA300 in

350 Pennsylvania region of the US and its subsequent range expansion was recently investigated in 
351 detail using genome sequences from 357 isolates from 22 states and territories and seven other

352 countries [51]. USA300 is now common internationally. Fluit and colleagues compared one 353 well-characterised strain from the US with those from Europe [52]. There was a difference of 354144 SNPs between the US isolate and those from Europe, the gene content showed 21 regions of 355 difference, and the European strains were resistant to fewer antibiotics. However, the SNP data 356 suggested a common ancestor around two decades ago [52]. This clone is an important pathogen 357 internationally, even if not as common in Europe as in the US, but that could change with spread 358 via ongoing international travel.

An outbreak of ST97-IVa involving 25 patients, originating from a surgical ward, over a four361 year period in Denmark, a country with a low prevalence of MRSA, was investigated by WGS 362 [53]. Eighteen patients had been admitted to the surgical ward of which 13 overlapped in terms 363 of admission periods. Two HCWs and two patient family members were also involved in the 364 outbreak. All except two isolates were spa type t267 and belonged to ST97. In this outbreak, 365 WGS linked nine initial isolates to 16 previous isolates, resulting in 23 patients being involved 366 with the suggestion that a healthcare worker with undetected carriage may have caused the 367 outbreak [53]. The authors noted that with shorter lengths of stay, patients may not be identified 368 as being colonized with MRSA carriers while in hospital but on follow up in the community after 369 discharge from hospital [53].

371 Modern healthcare requires patients to be transferred between hospitals as specialist and tertiary 372 facilities are centralised. A comparison of EMRSA-15 within the UK and Ireland using WGS 373 showed that the hospitals within the same referral regions had similar MRSA populations but 
374 transmission within a hospital arose from patients having been transferred from another hospital

375 [54]. Furthermore, frequent patient admissions to multiple hospitals results in ward-based

376 transmission within a hospital, as detected by a study in two NHS hospital groups and a district

377 general hospital in South-East London, involving ST22 MRSA isolates [55].

378

379 As the age of the population increases, patients are discharged more quickly than before from 380 acute hospitals to LSRU, such as to nursing homes. Sometimes these patients require re381 admission and there is constant flux between the acute and long-stay sector. Many studies have 382 highlighted this dynamic in terms of the acquisition and transmission of MRSA between these 383 sectors [56-62]. Furthermore, there has been interest in the role of carriage, including enteric 384 carriage [44,50]. In a long-term outbreak involving 1,600 patients and where WGS was used to 385 determine the origin, a single clonal variant of ST228 was responsible but that this clone was 386 more frequently recovered from the groin and rectal swabs [50].

In Singapore, where 1700 hospital patients and LSRU occupants were screened for MRSA over 389 a 6-week period, MRSA prevalence was lowest in acute healthcare facilities, i.e. 11.8\% 390 compared with intermediate or long-term care facilities, 29.95 and $20.4 \%$, respectively [56].

391 Furthermore, LSRUs had the greatest diversity of MRSA clones. Stine and colleagues examined 392 the transmission of MRSA from resident-to-resident in LSRUs using WGS. Multiple sites from 393 residents were screened over a 12-week study [59]. Isolates from multiple body sites were 394 usually closely related and many residents living together often harboured closely related strains $395[59]$. 
397 Following a protracted hospital outbreak between June 2013 and June 2016 caused by multidrug resistant ST1-MRSA-IV isolates belonging to spa type t157, a collection of 89 isolates from the outbreak hospital, 16 other hospitals and four other healthcare facilities and the community in Ireland were investigated by WGS [61]. Fifty of the isolates including 40 from the outbreak exhibited high-level mupirocin resistance mediated by a iles2-encoding plasmid conjugative 402 plasmid [61]. Pairwise SNVs exhibited by healthcare-associated and community-associated 403 isolates indicated recent transmission of ST1-MRSA-IV within and between multiple hospitals, 404 healthcare facilities and communities in Ireland [60]. This has implications for current MRSA 405 prevention and control guidelines, which are very much focused on measures in the acute 406 hospital sector. More recent studies have identified the multidrug resistant ST1-MRSA-IV clone 407 as a novel CC1-MRSA-IV clone that has recently emerged in several European countries (see 408 section on the identification of emerging MRSA clones below).

When developing guidelines, the question of what measures to take within households where 411 there is an MRSA-positive individual, often arises. Current Irish recommendations are to 412 highlight personal standards of hygiene and cleanliness and to minimise 413 disruption/inconvenience in a home, as intra-familial spread is not considered common [63]. A 414 retrospective study of isolates collected between 2008 and 2010 in Chicago and Los Angeles 415 found very little genetic variation amongst USA300 isolates within households but that 416 transmission did occur where the index patient had skin and soft tissue infection [64]. In 417 England, where USA300 is less common than in the United States, a 12-month prospective 418 observational study of 2283 screening and clinical isolates from 1465 patients collected between 419 April 2012 and April 2013 from the community and hospitals, found USA300 in only 24 cases 
$420 \quad(1.6 \%)$. There were also three groups of closely related isolates with a maximum genetic distance

421 of 6, 59 and 9 SNPs, respectively, amongst epidemiologically linked cases [65]. The authors

422 concluded that international travel may have played a role in the introduction of this clone into

423 England.

424

425 Over the last decade, it has been increasingly recognised that some patients without a recent 426 history of healthcare contact who develop MRSA may have done so via contact with livestock, 427 i.e. livestock-associated MRSA (LAMRSA). This has been particularly well described in those 428 countries with a low background prevalence of hospital MRSA such as Denmark. Larsen and 429 colleagues analysed human cases of LA-MRSA during 2010-2015 in Denmark [66]. Seventeen 430 cases of BSI, 700 cases of skin and soft tissue infection and 76 cases of other infections due to 431 LAMRSA, were studied. Overall, 32\% of the LAMRSA were from individuals with no contact 432 with livestock. Whole-genomes sequence analysis suggested that most isolates were closely 433 related to Danish pig isolates [66]. A study of CC398, commonly LAMRSA, was assessed in 434 human and pig isolates in Norway [67]. A human case was identified in 2009 but by the end of 435 2014, there were a total of 84 human cases. Epidemiological links placed these individuals in 436 three clusters and all farms had farm workers originating from other European countries where 437 MRSA is more prevalent [67]. Interestingly, while the farm workers and other possible human 438 carriers may have been non-Norwegian, none of the farms had imported pigs from abroad, and 439 the transmission of this clonal complex may have been by human introduction via migration 440 rather than through the importation of pigs. The results of this study would seem to justify the 441 Norwegian control strategy of targeting the screening of personnel before working in pig herds 
442 as part of national surveillance [65]. Contact with livestock is therefore a potential risk factor to

443 be considered and not just in countries with a low prevalence of MRSA in acute hospitals.

445 National surveillance systems together with key performance indicators increasingly drive 446 reductions in serious infections due to MRSA, particularly BSI. In the UK, a "zero tolerance" 447 approach has been advocated but there may be a portion of MRSA BSIs that are not preventable.

448 In one instance, WGS assisted in determining if an outbreak was preventable through infection 449 prevention and control measures. A study in Cambridge, UK, focussed on a cluster of five 450 MRSA BSIs between September 2011 and August 2012. The researchers used detailed 451 epidemiological methods and WGS analysis of isolates and found that there were varying 452 degrees of overlap in admission to the wards of these cases [68]. A comparison of isolates 453 indicated that each patient was infected by their own carriage isolate. Amongst four of the 454 patients, isolates differed by between 122 and 168 cgSNPs [68]. A wider analysis of all patients 455 with MRSA, whether colonised or infected was undertaken including the cases of MRSA BSIs.

456 Three of the five BSI episodes were associated with skin conditions and two were attributable to 457 intravascular catheters. From an analysis of the WGS data, it was concluded that these cases 458 were not due to inter-patient transmission [68]. In this setting, WGS suggested that there was no 459 deficiency of infection prevention and control services in preventing these five cases of BSI, and 460 that some or all these cases of BSI were probably not preventable.

\section{Identification of emerging MRSA clones}

463 A study by Earls et al. (2019) used WGS to investigate the recent emergence of multidrug464 resistant Panton-Valentine leukocidin (PVL)-negative CC1-MRSA-IV isolates in multiple Irish 
465 hospitals and the community and in two hospitals in the German City of Regensburg between 466 2016-2018 where it was also identified in the community [69]. Phylogenetic analysis grouped the

467 isolates into a large clade, where no isolate differed from any other isolate by more than 130 468 cgSNVs. Clade isolates harboured an SCCmec type IVa element with a characteristic 4710 469 nucleotide insertion in the downstream constant segment $(d c s)$ adjacent to orf $X$ and harboured 470 the same allelic variants of the SCCmec genes, ccrA2 (1350 bp) and ccrB2 (1629 bp). Overall, 471 clade isolates exhibited genotypic characteristics which differed comprehensively from those 472 associated with other previously well characterised CC1-MRSA-IV clones including Western 473 Australia (WA) MRSA-1 and USA400. Five MRSA isolates recovered in a Romanian hospital 474 between 2010 and 2012 and 10 CC1 MSSA isolates recovered in the same Romanian hospital 475 between 2009 and 2012 also grouped into the novel CC1 clade. Earls et al. (2019) designated the 476 multidrug resistant MRSA isolates as a novel European clade of CC1-MRSA-IV and 477 hypothesized that this clade likely recently emerged from CC1 MSSA in Romania or a 478 neighbouring country [69]. Interestingly, isolates of this emerging European CC1-MRSA-IV 479 clone were also recently identified in an Italian paediatric hospital [24].

\section{Routine applications}

482 Having data interpreted within two to three days or less, will enhance infection prevention and 483 control measures and may prevent the use of disruptive measures such as the closing of units or 484 wards. Eyre and colleagues used rapid benchtop sequencing to investigate two outbreaks of $S$. 485 aureus within five days of a positive culture result [70]. One involved ten MRSA isolates from 486 eight patients in an intensive care unit and the other involved six patients over three months in 487 the south of England with Panton-Valentine leucocidin positive MRSA. Within clusters or 
488 outbreaks, most isolates were indistinguishable, and all were within three SNVs [70]. On a

489 broader public health front, WGS may be used to track community and hospital-acquired isolates

490 within and between countries. A total of 308 invasive isolates collected across Europe identified

491 predominant clones, e.g. clonal complexes 5 and 22, with the latter representing EMRSA-15

492 originally from the UK [71].

493

494 Conclusions

495 The application of WGS has greatly expanded our knowledge of clinical and epidemiologic 496 aspects of MRSA infection and colonization, including transmission, the commonality of clones

497 in the community as well as the evolution of resistant determinants. Subsequent studies will 498 further inform our understanding of transmission patterns and guide what interventions are most 499 important and when they should be applied. The use of WGS in day-to-day practice will be 500 facilitated by improvements in our capacity to interpret the data and apply it appropriately, in a 501 timely manner. This may in many instances confirm low level but ongoing clusters and cross502 transmission of MRSA, which if acted on, may assist in preventing larger outbreaks. The 503 availability of WGS data may also suggest considerable patient-to-patient transmission of a 504 single clone or that an outbreak is due to the simultaneous emergence of different clones even if 505 patients are temporally and geographically linked. Alternatively, an outbreak may arise from 506 MRSA being carried into a hospital, from either other hospitals or a LSRU. 
510 Future priorities include agreeing parameters for deciding whether isolates are closely related or 511 otherwise enhanced data analysis and ensuring IT systems can share WGS data between 512 hospitals, between countries and further afield. As this field continues to mature and evolve, 513 WGS may potentially have a significant role in informing measures to prevent transmission 514 through the provision of critically important molecular epidemiological information in real time. 
517 Declaration: $\mathrm{HH}$ has been in receipt of research funding from Pfizer and Astellas and has 518 provided educational sessions or advice to Pfizer and Cepheid in recent years.

519 Funding: The preparation and drafting of this review was not supported by any external funding. 520 However, the authors would like to acknowledge recent research funding from the Irish Health 521 Research Board (grant number HRA-POR-2015-1051) and previously from Scientific 522 Foundation Ireland for research on MRSA in recent years. We also acknowledge and thank Paul 523 J Murphy for assistance in the literature search. 


\section{References}

1. Zingg W, Hopkins S, Gayet-Ageron A, Holmes A, Sharland M, Suetens C for the ECDC PPS study group. Health-care-associated infections in neonates, children, and adolescents: an analysis of paediatric data from the European Centre for Disease Prevention and Control point-prevalence survey. Lancet Infect Dis 2017; 17: 381-89.

2. Smyth ETM, McIlvenny G, Enstone JE, Emmerson AM, Humphreys H, Fitzpatrick F, et al. Four country healthcare associated infection prevalence survey 2006: overview of the results. J Hosp Infect 2008; 69: 230-248.

3. Guy R, Geoghegan L, Heginbothom M, Howe R, Muller-Pebody B, Reilly JS, et al. Nonsusceptibility of Eschericihia coli., Klebsiella spp., Pseudomonas spp., Streptococcus pneumoniae and Staphylococcus aureus in the UK: temporal trends in England, Northern Ireland, Scotland and Wales. J Antimicrob Chemother 2016; 71: 1564-1569.

4. Mizuno S, Iwami M, Kunisawa S, Naylor N, Yamashita K, Kyratsis Y, et al. Comparison of national strategies to reduce meticillin-resistant Staphylococcus aureus infections in Japan and England. J Hosp Infect 2018; 100: 280-298.

5. Monecke S, Slickers P Ehricht R. Assignment of Staphylococcus aureus isolates to clonal complexes based on microarray analysis and pattern recognition. FEMS Immunol Med Microbiol 2008; 53: 237-51.

6. Monecke S, Jatzwauk L, Müller E, Nitschke H, Pfohl K, Slickers P, et al. Diversity of SCCmec elements in Staphylococcus aureus as observed in south-eastern Germany. PLoS One 2016; 11(9): e0162654. doi: 10.1371/journal.pone.0162654.

7. Shore AC, Rossney AS, Kinnevey PM, Brennan OM, Creamer E, Sherlock O, Dolan A, et al. Enhanced discrimination of highly clonal ST22-methicillin-resistant Staphylococcus aureus IV isolates achieved by combining spa, dru and pulsed-field gel electrophoresis typing data. J Clin Microbiol 2010; 48:1839-52.

8. Holden MT, Hsu LY, Kurt K, Weinert LA, Mather AE, Harris SR, et al. A genomic portrait of the emergence, evolution, and global spread of a methicillin-resistant Staphylococcus aureus pandemic. Genome Res 2013; 23:653-64.

9. Grundmann H, Schouls LM, Aanensen DM, Pluister GN, Tami A, Chlebowicz M, et al. ESCMID Study Group on Molecular Epidemiological Markers, European Staphylococcal Reference Laboratory Working Group. The dynamic changes of dominant clones of Staphylococcus aureus causing bloodstream infections in the European region: results of a second structured survey. Euro Surveill 2014; 19(49):pii_20987. http://www.eurosurveillance.org/ViewArticle.aspx?ArticleId_20987.

10. Creamer E, Shore AC, Rossney AS, Dolan A, Sherlock O, Fitzgerald-Hughes D, et al. Transmission of endemic ST22-MRSA-IV on four acute hospital wards investigated using a combination of $s p a, d r u$, and pulsed-field gel electrophoresis typing. Eur J Clin Microbiol Infect Dis 2012; 31: 3151-3161.

11. Kinnevey PM, Shore AC, MacAogáin M, Creamer E, Brennan GI, Humphreys H, et al. Enhanced tracking of nosocomial transmission of endemic sequence Type 22 methicillinresistant Staphylococcus aureus type IV isolates among patients and environmental sites by use of whole-genome sequencing. J Clin Microbiol 2016; 54: 445-448.

12. Tang P, Croxen MA, Hasan MR, Hsiao WW, Hoang LM. Infection control in the new age of genomic epidemiology. Am J Infect Control 2017; 45:170-9. 
13. Besser J, Carleton HA, Gerner-Smidt P, Lindsey RL, Trees E. Next-generation sequencing technologies and their application to the study and control of bacterial infections. Clin Microbiol Infect 2018; 24:335-41.

14. Schürch AC, Arredondo-Alonso S, Willems RJL, Goering RV. Whole genome sequencing options for bacterial strain typing and epidemiologic analysis based on single nucleotide polymorphism versus gene-by-gene-based approaches. Clin Microbiol Infect 2018; 24:350 4.

15. Peacock SJ, Parkhill J, Brown NM. Changing the paradigm for hospital outbreak detection by leading with genomic surveillance of nosocomial pathogens. Microbiol 2018; 164:1213-9

16. Cunningham SA, Chia N, Jeraldo PR, Quest DJ, Johnson JA, Boxrud DJ, et al. Comparison of whole-genome sequencing methods for analysis of three methicillin-resistant Staphylococcus aureus outbreaks. J Clin Microbiol 2017; 55: 1946-1953

17. Leggett RM, Clark MD. A world of opportunities with nanopore sequencing. J Exp Bot 2017; 68:5419-29.

18. Lemon JK, Khil PP, Frank KM, Dekker JP. Rapid nanopore sequencing of plasmids and resistance gene detection in clinical isolates. J Clin Microbiol 2017; 55:3530-43.

19. Baig S, Johannesen TB, Overballe-Petersen S, Larsen J, Larsen AR, Stegger M. Novel SCCmec type XIII (9A) identified in an ST152 methicillin-resistant Staphylococcus aureus. Infect Genet Evol 2018; 61:74-6.

20. Carriço JA, Rossi M, Moran-Gilad J, Van Domselaar G, Ramirez M. A primer on microbial bioinformatics for nonbioinformaticians. Clin Microbiol Infect 2018; 24:342-9.

21. Oakeson KF, Wagner JM, Mendenhall M, Rohrwasser A, Atkinson-Dunn R. Bioinformatic analyses of whole-genome sequence data in a public health laboratory. Emer Infect Dis 2017; 23:1441-5.

22. Tong SY, Holden MT, Nickerson EK, Cooper BS, Köser CU, Cori A, et al. Genome sequencing defines phylogeny and spread of methicillin-resistant Staphylococcus aureus in a high transmission setting. Genome Res 2015; 25:111-8.

23. Gordon NC, Pichon B, Golubchik T, Wilson DJ, Paul J, Blanc DS, et al. Whole-genome sequencing reveals the contribution of long-term carriers in Staphylococcus aureus outbreak investigation. J Clin Microbiol 2017; 55:2188-97.

24. Manara S, Pasolli E, Dolce D, Ravenni N, Campana S, Armanini F, et al. Whole-genome epidemiology, characterisation, and phylogenetic reconstruction of Staphylococcus aureus strains in a paediatric hospital. Genome Med 2018; 10(1):82 doi: 10.1186/s13073-018-05937.

25. Enright MC, Day NPJ, Davies CE, Peacok SJ, Spratt BG. Sequence typing for characterization of methicillin-resistant and methicillin-susceptible clones of Staphylococcus aureus. J Clin Microbiol 2000;38: 1008-1015.

26. Leopold SR, Goering RV, Witten A Harmsen D, Mellmann A. Bacterial whole-genome sequencing revisited: portable, scalable, and standardized analysis for typing and detection of virulence and antibiotic resistance genes. J. Clin. Microbiol 2014, 52, 2365-70.

27. Roisin S, Gaudin C, De Mendonça R, Bellon J, Van Vaerenbergh K, De Bruyne K. et al. Pan-genome multilocus sequence typing and outbreak-specific reference-based single nucleotide polymorphism analysis to resolve two concurrent Staphylococcus aureus outbreaks in neonatal services. Clin Microbiol Infect 2016; 22:520-6.

28. Gilchrist CA, Turner SD, Riley MF, Petri Jr WA, Hewlett EL. Whole-genome sequencing in outbreak analysis. Clin Microbiol Rev 2015; 28: 541-563. 
29. Croucher NJ, Didelot X. The application of genomics to tracing bacterial pathogen transmission. Curr Opin Microbiol. 2015; 23:62-7.

30. Bartels MD, Petersen A, Worning P, Nielsen JB, Larner-Svensson H, Johansen HK, et al. Comparing whole-genome sequencing with Sanger sequencing for spa typing of methicillinresistant Staphylococcus aureus. J Clin Microbiol 2014; 52: 4305-4308.

31. Quainoo S, Coolen JPM, van Hijum SAFT, Huynen MA, Melchers WJG, van Schaik W, Wertheim HFL. Whole-genome sequencing of bacterial pathogens: the future of nosocomial outbreak analysis. Clin Microbiol Rev 2017; 30:1015-1063.

32. Harris SR, Feil EJ, Holden MTG, Quail MA, Nickerson EK, Chantratita N et al. Evolution of MRSA during hospital transmission and intercontinental spread. Science 2010; 327: 469474.

33. Price JR, Didelot X, Crook DW, Llewelyn MJ, Paul J. Whole genome sequencing in the prevention and control of Staphylococcsu aureus infection. J Hosp Infect 2013; 83: 14-21.

34. Young BC, Golubchik T, Batty EM, Fung R, Larner-Svensson H, Votintseva AA, et al. Evolutionary dynamics of Staphylococcus aureus during progression from carriage to disease. PNAS 2012; 109: 4550-4555.

35. Nübel U, Nachtnebel M, Falkenhorst G, et al. MRSA Transmission on a neonatal intensive care unit: epidemiological and genome-based phylogenetic analyses. PLoS 2013; 8(1): e54898. doi: 10.137/jopurnal.pone.0044898

36. Layer F, Sanchini A, Strommenger B, Cuny C, Breier A-C, Proquitté H, et al. Molecular typing of toxic shock syndrome toxin-1- and enterotoxin A-producing methicillin-sensitive Staphylococcus aureus isolates from an outbreak in a neonatal intensive care unit. Internat $\mathbf{J}$ Med Micro 2015; 305: 790-798.

37. Köser CU, Holden MTG, Ellington MJ,Ellington MJ, Cartwright EJP, Brown NM, et al. Rapid whole-genome sequencing for investigation of a neonatal MRSA outbreak. N Engl Med 2012; 366: 2267-75.

38. Harris SR, Cartwright EJP, Török ME, Holden MTG, Brown NM, Ogilvy-Stuart AL, et al. Whole-genome sequencing for analysis of an outbreak of methicillin-resistant Staphylococcus aureus; a descriptive study. Lancet Infect Dis 2013; 13: 130-136.

39. Azaraian T, Maraqa NF, Cook RL, Johnson JA, Bailey C, Wheeler S, et al. Genomic epidemiology of methicillin-resistant Staphylococcus aureus in a neonatal intensive care unit. PLoS One 2016; 11(10): e0164397. doi:10.371/journal.pone.0164397.

40. Azarian T, Cook RI, Johnson JA, Guzman N, McCarter YS, Gomez N, et al. Whole-genome sequencing for outbreak investigations of methicillin-resistant Staphylococcus aureus in the neonatal intensive care unit: time for routine practice? Infect Control Hosp Epidemiol 2015; 36(7): 777-785.

41. Ugolotti E, Di Marco E, Bandettini R, Biassoni R. Genomic characterisation of a paediatric MRSA outbreak by next-generation sequencing. J Hosp Infect 2018; 98: 155-160.

42. Earls MR, Coleman DC, Brennan GI, Fleming T, Monecke S, Slickers P, et al. Intra-hospital, inter-hospital and intercontinental spread of ST78 MRSA from two neonatal intensive care units outbreaks established using whole genome sequencing. Front Microbiol 2018; 9.1485.doi: 10.3389/fmicb.2018.01485.

43. Durand G, Javerliat F, Bes M, Veyrieras J-B, Guigon G, Mugnier N, et al. Routine wholegenome sequencing for outbreak investigations of Staphylococcus aureus in a national reference center. Front Microbiol 2018; 9.511. doi: 10.3389/fmicb.2018.00511. 
44. Kong Z, Zhao P, Liu H, Yu X, Qin Y, Su Z, et al. Whole-genome sequencing for the investigation of a hospital outbreak of MRSA in China. PLoS ONE 2016; 11(3): e0149844, doi: 10.1371/journal.pone.0149844.

45. Price JR, Cole K, Bexley A, Kostiou V, Eyre DW, Golubchik T, et al. Transmission of Staphylococcus aureus between health-care workers, the environment, and patients in an intensive care unit: a longitudinal cohort study based on whole-genome sequencing. Lancet Infect Dis 2017; 17: 207-14.

46. Weterings V, Bosch T, Witteveen S, Landman F, Schouls L, Klutyman J. Next-generation sequence analysis reveals transfer of methicillin resistance to a methicillin-susceptible Staphylococcus aureus strain that subsequently caused a methicillin-resistant Staphylococcus aureus outbreak: A descriptive study. J Clin Microbiol 2017; 55: 2808-2816.

47. Long WS, Beres S, Olsen RJ, Musser JM. Absence of patient-to-patient intra-hospital transmission of Staphylococcus aureus as determined by whole-genome sequencing. mBio 2014; 5(5): e01692-14.doi.10.1128/mbio.01692-14.

48. Miller RR, Price JR, Batty EM et al. Healthcare-associated outbreak of methicillin-resistant Staphylococcus aureus bacteraemia: role of a cryptic variant of an epidemic clone. J Hosp Infect 2014; 86: 83-89.

49. Moore G, Cookson B, Gordon NC, Jackson R, Kearns A, Singleton J, et al. Whole-genome sequencing in hierarchy with pulsed-field gel electrophoresis: the utility of this approach to establish possible sources of MRSA cross-transmission. J Hosp Infect 2015; 90: 38-45.

50. Senn L, Clerc O, Zanetti G, Bassett P, Prod'hom G, Gordon NC, et al. The stealthy superbug: The role of asymptomatic enteric carriage in maintaining a long-term hospital outbreak of ST228 methicillin-resistant Staphylococcus aureus. mBio 2016; 7(1): e0203915.doi.10.1128/mBio.02039-15.

51. Challagundla L, Luo X, Tickler IA, Didelot X, Coleman DC, Shore AC, et al. Range expansion and the origin of USA300 North American epidemic methicillin-resistant Staphylococcus aureus. MBio 2018; 9(1):e02016-17. doi: 10.1128/mBio.02016-17.

52. Fluit, AC, Carpaij N, Majoor EAM, Weinstein RA, Aroutcheva A, Rice TW, et al. Comparison of an ST80 MRSA strain from the USA with European ST80 strains. J Antimicrob Chemother 2015; 70: 664-669.

53. Rubin IM, Hansen TA, Klingenberg AM, Petersen AM, Worning P, Westh H, et al. A sporadic four-year hospital outbreak of a ST97-IVa MRSA with half of the patients identified in the community. Front Microbiol 2018; 9.1494. doi: 10.3389/fmicb.2018.0194.

54. Donker, T, Reuter S, Scriberras J,Reynolds R, Brown NM, Török ME et al. Population genetic structuring of methicillin-resistant Staphylococcus aureus clone EMRSA-15 within UK reflects patient referral patterns. Microbial Genom 2017; 3: e000113. Doi.10.1099/mgen.0.000113.

55. Auguet, OT, Stabler RA, Betley J, Preston MD, Dhaliwal M, Ioannou A, et al. Frequent undetected methicillin-resistant Staphylococcus aureus ward-based; transmission linked to patient sharing between hospitals. Clin Infect Dis 2018; 66: 840-8.

56. Chow A, Lim VW, Khan A, Pettigrew K, Lye DCB, Kanagasabai K, et al. MRSA transmission dynamics among interconnected acute, intermediate-term, and long-term healthcare facilities in Singapore. Clin Infect Dis 2017: 64: S76-S81.

57. Toleman, MS, Watkins ER, Williams T, Blane B, Sadler B, Harrison EM et al. Investigation of a cluster of sequence type 22 methicillin-resistant Staphylococcus aureus transmission in a community setting. Clin Infect Dis 2017; 65: 2069-2077. 
58. de la Gandara MP, Garay JAR, Mwangi M, Tobin JM, Tsang A, Khalida C, et al. Molecular types of methicillin-resistant Staphylococcus aureus and methicillin-sensitive $S$. aureus strains causing skin and soft tissue infections and nasal colonization, identified in community health centers in New York City. J Clin Microbiol 2015; 53: 2648-2658.

59. Stine, OC, Burrowes S, David S, Johnson JK, Roghmann M-C. Transmission clusters of methicillin-resistant Staphylococcus aureus in long-term care facilities based on wholegenome sequencing. Infect Control Hosp Epidemiol 2016; 37: 685-691.

60. Auguet TO, Betley JP, Stabler RA. Evidence for community transmission of communityassociated but not health-care-associated methicillin-resistant Staphylococcus aureus strains linked to social and material deprivation: spatial analysis of cross-sectional data. PLoS Med 2016: 13. Doi.10.1371/journal.pmed.1001944.

61. Earls MR, Kinnevey PM, Brennan GI, Lazaris A, Skally M, O’Connell B, et al. The recent emergence in hospitals of multidrug-resistant community-associated sequence type 1 and spa type 127 methicillin-resistant Staphylococcus aureus investigated by whole-genome sequencing: Implications for screening. PLoS ONE 2017: 12; e0175542.foi.10.1371/jouranl.pone.0175542.

62. Harrison EM, Zudden C, Broderick HJ, Blane B, Brennan G, Morris D et al. Transmission of methicillin-resistant Staphylococcus aureus in long-term care facilities and their related healthcare networks. Genome Med 2016; 8: doi 10.1186/s13073-016-0353-5.

63. National Clinical Effectiveness Committee. Prevention and control of methicillin-resistant Staphylococcus aureus. National Clinical Guideline No.2. Department of Health, Dublin, Ireland, September 2013. http://www.hpsc.ie/az/microbiologyantimicrobialresistance/infectioncontrolandhai/guidelines/File, 14478,en.pdf (accessed $26^{\mathrm{th}}$ January 2019).

64. Alam MT, Read TD, Petit III RA, Petit III RA, Boyle-Vavra S, Miller LG et al. Transmission and microevolution of USA300 MRSA in U.S. households: Evidence from whole-genome sequencing. mBio 2015; 6(2): e000-54-15. Doi.10.1128/mBio.00054-15.

65. Toleman MS, Reuter S, Coll F, Harrison EM, Blane B, Brown NM, et al. Systematic surveillance detects multiple silent introductions and household transmission of methicillinresistant Staphylococcus aureus USA 300 in the East of England. J Infect Dis 2016; 214: 447-453.

66. Larsen J, Peterson A, Larsen AR, Sieber RN, Stegger M, Koch A, et al. Emergence of livestock-associated methicillin-resistant Staphylococcus aureus bloodstream Infections in Denmark. Clin Infect Dis 2017: 65: 1072-1076.

67. Grøntvedt, CA, Elstrøm P, Stegger M, Skov RL, Andersen PS, LArssen KW, et al. Methicillin-resistant Staphylococcus aureus CC398 in humans and pigs in Norway: A "One Health" perspective on introduction and transmission. Clin Infect Dis 2016; 63: 1431-1438.

68. Török, ME, Harris SR, Cartwright EJP, Raven KE, Brown NM, Allison MED, et al. Zero tolerance for healthcare-associated MRSA bacteraemia: is it realistic? J Antimicrob Chemother 2014; 69: 2238-2245.

69. Eyre DW, Golubchik T, Gordon NC, Bowden R, Piazza P, Batty EM, et al. A pilot study of rapid benchtop sequencing of Staphylococcus aureus and Clostridium difficile for outbreak detection and surveillance. BMJOpen 2012; 2: e001124.doi:10.1136/bmjopen-20120011124. 
70. Earls MR, Shore AC, Brennan GI, Simbeck A, Brachert WS, Vremeră T, et al. A novel multi-resistant PVL-negative CC1-MRSA-IV clone emerging in Ireland and Germany likely evolved in South-Eastern Europe. Infect Genet Evol 2019; 69:117-126.

71. Aanensen DM, Feil EJ, Holden MTG, Dordel J, Yeats CA, Fedosejev A, et al. Wholegenome sequencing for routine pathogen surveillance in public health; a population snapshot of invasive Staphylococcus aureus in Europe. mBio 2016; 7(3): e0044416.doi:10.1128/mbio.oo4444-16. 
Table I. List of commonly used terms relating to whole genome sequencing and analysis

\begin{tabular}{|c|c|}
\hline Term & Explanation \\
\hline Next generation sequencing (NGS) & $\begin{array}{l}\text { The high-throughput and rapid determination of } \\
\text { whole genome sequences. Millions to billions of } \\
\text { DNA fragments are sequenced in parallel. }\end{array}$ \\
\hline Sequencing platform & $\begin{array}{l}\text { DNA squencing system consisting of sequencing } \\
\text { equipment (i.e. sequencer) and methodology to } \\
\text { sequence target DNA. Examples include the } \\
\text { Illumina MiSeq, the PacBio Single Molecular } \\
\text { Real-Time (SMRT) and the Oxford Nanopore } \\
\text { MinION sequencing platforms }\end{array}$ \\
\hline Sequence read & $\begin{array}{l}\text { A continuous DNA sequence determined from a } \\
\text { target organism (e.g. bacterium) }\end{array}$ \\
\hline Short sequence reads & $\begin{array}{l}\text { A continuous DNA sequence determined from a } \\
\text { target organism ranging between approximately } \\
100-600 \mathrm{bp} \text {. For example, the Illumina MiSeq } \\
\text { sequencing platform yields short sequence reads }\end{array}$ \\
\hline Long sequence reads & $\begin{array}{l}\text { A continuous DNA sequence determined from a } \\
\text { target organism generally }>10 \mathrm{~kb} \text {. For example, } \\
\text { the PacBio SMRT sequencing platform yields } \\
\text { long sequence reads }\end{array}$ \\
\hline Sequence read error rate & $\begin{array}{l}\text { The proportion of sequence reads containing } \\
\text { sequencing errors }\end{array}$ \\
\hline Contigs & $\begin{array}{l}\text { Contiguous sequences assembled from } \\
\text { overlapping smaller sequence reads that represent } \\
\text { a consensus region of DNA }\end{array}$ \\
\hline Single nucleotide variation (SNV) & $\begin{array}{l}\text { A variation in a single nucleotide base that occurs } \\
\text { at a specific position in the genome of an } \\
\text { organism of interest without implying how often } \\
\text { this variation occurs in a population }\end{array}$ \\
\hline Single nucleotide polymorphism (SNP) & $\begin{array}{l}\text { A variation in a single nucleotide base that occurs } \\
\text { at a specific position in the genome of an } \\
\text { organism of interest and is relatively common in } \\
\text { a population }\end{array}$ \\
\hline SNP analysis & $\begin{array}{l}\text { Strain typing by mapping SNPs in sequence reads } \\
\text { or assembled contigs against a reference } \\
\text { genome(s). Reference genomes that are closely } \\
\text { related to the sequenced samples are best }\end{array}$ \\
\hline Core genome & $\begin{array}{l}\text { A set of conserved genes present in virtually all } \\
\text { isolates of a species. }\end{array}$ \\
\hline Accessory genome & $\begin{array}{l}\text { A variable set of genes present in some but not all } \\
\text { isolates of a species. Examples in } S \text {. aureus and } \\
\text { other staphylococci include SCCmec and arginine } \\
\text { catabolic mobile elements (ACME) }\end{array}$ \\
\hline $\begin{array}{l}\text { Multilocus sequence typing (MLST). } \\
\text { Also known as conventional MLST }\end{array}$ & $\begin{array}{l}\text { A method that determines the sequences of } \\
\text { internal fragments of up to seven housekeeping } \\
\text { genes. The different sequences within a bacterial }\end{array}$ \\
\hline
\end{tabular}




\begin{tabular}{|c|c|}
\hline $\begin{array}{l}\text { Multilocus sequence typing (MLST). } \\
\text { Also known as conventional MLST }\end{array}$ & $\begin{array}{l}\text { A method that determines the sequences of } \\
\text { internal fragments of up to seven housekeeping } \\
\text { genes. The different sequences within a bacterial } \\
\text { species are assigned as distinct alleles and, for } \\
\text { each isolate, the alleles at each of the loci define } \\
\text { the allelic profile or sequence type (ST). Curated } \\
\text { MLST databases exist for many bacterial species } \\
\text { including } S \text {. aureus. Conventional MLST has } \\
\text { largely been replaced by WGS-based MLST } \\
\text { schemes for S. aureus and other important } \\
\text { bacterial pathogens due to significantly enhanced } \\
\text { resolution }\end{array}$ \\
\hline Whole genome MLST (wgMLST). & $\begin{array}{l}\text { A typing method that uses WGS data from the } \\
\text { core genome and accessory genome to perform } \\
\text { MLST on a genome-wide basis and permits gene-- } \\
\text { by gene comparisons of very large numbers of } \\
\text { genes amongst a group of isolates. The } \\
\text { presence/absence of each target locus is } \\
\text { determined from WGS data, as are allelic } \\
\text { variants. Very high resolution can be achieved }\end{array}$ \\
\hline Core genome MLST (cgMLST) & $\begin{array}{l}\text { A typing method that uses WGS data to perform } \\
\text { MLST on a predefined set of conserved genome- } \\
\text { wide core (can be }>1000 \text { genes) that are present } \\
\text { in the vast majority of isolates of a bacterial } \\
\text { species. Very high resolution can be achieved } \\
\text { across large groups of isolates by core genome } \\
\text { comparisons. The approach is highly } \\
\text { reproducible across data sets }\end{array}$ \\
\hline Genome assembly & $\begin{array}{l}\text { The entire DNA sequence of an organism can be } \\
\text { reassembled from overlapping sequence reads } \\
\text { which first are assembled into larger contigs. Any } \\
\text { remaining gaps between contigs can then be }\end{array}$ \\
\hline
\end{tabular}


Table II. Studies using whole genomic sequencing (WGS) of MRSA in the neonatal/paediatric setting

\begin{tabular}{ll}
\hline $\begin{array}{l}\text { Country } \\
\text { (Ref) }\end{array}$ & Study Details \\
\hline $\begin{array}{l}\text { Germany } \\
(35)\end{array}$ & $\begin{array}{l}\text { Retrospective case control } \\
\text { study of neonates and staff }\end{array}$ \\
Germany & $\begin{array}{l}\text { Outbreak of toxic-shock } \\
\text { syndrome \& enterotoxin- } \\
\text { A producing MSSA }\end{array}$ \\
UK & $\begin{array}{l}\text { Retrospective } \\
\text { investigation of outbreak } \\
\text { in neonatal intensive care } \\
\text { unit }\end{array}$ \\
Outbreak in neonatal unit \\
(38) & $\begin{array}{l}\text { with isolates compared to } \\
\text { other clinical strains in the } \\
\text { hospital or community }\end{array}$ \\
&
\end{tabular}

USA Retrospective

(39) analysis of isolates from screening neonates, 20082010

\section{Main Conclusions}

28 isolates formed a predominant strain

with two other strains present

Staff involved in transmission

$26 \%$ of neonates positive; $19.5 \%$ of staff

21 related isolates confirming PFGE analysis

$2.8 \times 10^{-6}$ mutations per nucleotide per year

Distinct cluster of outbreak isolates and clear separation with non-outbreak isolates.

11 MRSA infants identified

ST2371 predominated

Similar isolates in emergency department, out-patients \& general practice

Colonised infants had lower birth weight, gestational age and length of stay

$70 \%$ of colonization due to events within the NICU. Transmission varied by strain

17 neonates acquired MRSA in unit

12 unique isolates with 5 in two clusters 300 identified on PFGE and analysed with WGS \& spa typing

Italy Isolates from putative

(41) outbreak in NICU \& PICU

Isolates from two

10/12 isolates ST625; 2xST8

A maximum $1.7 \%$ phylogenetic distance amongst ST625

A staff member isolated with ST625

Considerable homogeneity between 28

\section{Comment}

Only one isolate per neonate/staff analysed and greater variability may have been present

Prolonged duration of outbreak suggests that staff member may have been reservoir as far back as 2-3 years ago

Value of WGS in real time for MRSA control in hospitals

Mother-to-mother transmission outside hospital \& staff carriage allowed outbreak to persist

Useful longitudinal analysis of strains with evidence of significant within-unit spread and dynamic changes in strain predominance

WGS excluded cross-transmission in most cases, suggesting multiple and independent introduction of MRSA strains
Genetically related isolates but temporal analysis of isolates did not confirm an outbreak

Highlights the importance of travel in the 
Ireland

France outbreaks of MRSA ST78

in a neonatal intensive care unit

Isolates from two geographically separate NICUs isolates with likely importation from abroad and involvement of healthcare worker

$41 \mathrm{CC}$ isolates from Limoges and Bordeaux with less than 22 SNPs difference spread of MRSA and the value of WGS in tracking local spread and determining the origins of clones

WGS is useful to determine relatedness but also to track bacterial evolution

MSSA; methicillin-susceptibility Staphylococcus aureus, PFGE; pulsed-field gel electrophoresis, NICU, neonatal intensive care unit, PICU; paediatric intensive care unit, CC, clonal complexes, SNPs, short nucleotide polymorphism. 